\title{
TRATAMENTO VÍDEO ASSISTIDO DE INTUSSUSCEPÇÃO INTESTINAL POR ADENOCARCINOMA DE CECO EM CRIANÇA
}

\section{VIDEO ASSISTED TREATMENT OF INTESTINAL INTUSSUSCEPTION FOR CECUM ADENOCARCI- NOMA IN CHILDHOOD}

Loraine Entringer Falqueto ${ }^{1}$; Paula Rubio Vilar ${ }^{1}$; Carolina Martins Vissoci1 ${ }^{1}$; Fernando Antônio Bersani Amado $^{1}$; Claudio Schulz ${ }^{1}$; Elisângela de Mattos e Silva ${ }^{1}$.

\section{RESUMO}

Introdução: A intussuscepção intestinal é uma das principais causas de obstrução intestinal em crianças. Em pacientes acima de cinco anos, em geral, é secundária a lesões predominantemente benignas, entretanto, os tumores malignos intestinais também podem causar invaginação intestinal. $O$ adenocarcinoma de cólon é um dos cânceres mais frequentes em adultos, porém é raro em pacientes pediátricos. Os pacientes apresentam subtipos mais agressivos e em estágios mais avançados e tem sobrevida média de 26 meses. Relato do caso: Neste trabalho é relatado o caso de um paciente masculino com 12 anos de idade diagnosticado com invaginação íleo-ceco-cólica por adenocarcinoma de ceco. Conclusão: A invaginação intestinal em pacientes acima de cinco anos está fortemente associada a pontos patológicos de invaginação. Causas benignas são mais frequentes, porém, dada a agressividade do adenocarcinoma de cólon nas crianças e nos adolescentes, deve-se estar atento para os sintomas de alarme com alto índice de suspeição, permitindo o diagnóstico precoce e o tratamento oncológico adequado.

Palavras-chave: Neoplasias do Cólon. Laparoscopia. Oncologia Cirúrgica. Saúde da Criança. Obstrução Intestinal.

\begin{abstract}
Introduction: Intestinal intussusception is a major cause of intestinal obstruction in children. Although in patients older than five years it is usually secondary to benign lesions, malignant intestinal tumors can also present as intestinal invagination. Colon adenocarcinoma is one of the most common cancers in adults, whereas in pediatric patients it is rare. Pediatric patients have more aggressive subtypes with more advanced stages. The median survival is about of 26 months. Case report: This paper reports the case of a 12year-old male patient diagnosed with ileocecholic invagination due to cecum adenocarcinoma. Conclusion: Intestinal invagination in patients over five year-old is strongly associated with pathological conditions of invagination. Benign causes are more frequent, however, given the aggressiveness of the colon adenocarcinoma in children and adolescents, we should be aware of the symptoms of alarm with a high index of suspicion, allowing early diagnosis and cancer proper treatment.

Keywords: Colonic Neoplasms. Video-Assisted Surgery. Surgical Oncology. Pediatric Emergency Medicine. Intestinal Obstruction.
\end{abstract}

\section{INTRODUÇÃO}

Tumores malignos primários de cólon são neoplasias raras em pacientes pediátricos. Com base em dados americanos, a taxa de câncer colorretal (CCR) em pacientes menores de 15 anos foi 0,3 para 100 mil entre 2016 e 2018'. A apresentação clínica, assim como em adultos, é inespecífica, com dor abdominal, anemia e perda de peso. Em alguns casos pode mimetizar o quadro clínico de apendicite aguda ou abdome agudo obstrutivo, sendo frequente o diagnóstico como uma urgência pediátrica oncológica ${ }^{2}$.
As lesões malignas presentes no mesentério e no intestino, apesar de pouco frequentes, podem interferir no peristaltismo intestinal e desencadear a intussuscepção ${ }^{3}$. Em crianças acima de 5 anos, é esperado o achado desses pontos patológicos como causa de invaginação ${ }^{3}$. O cirurgião pediátrico deve ter alta suspeição clínica e cuidados específicos devem ser tomados para que seja realizado o melhor tratamento oncológico possivel.

Neste artigo, é relatada uma apresentação incomum de adenocarcinoma 
de cólon em adolescente como invaginação intestinal.

\section{RELATO DO CASO}

Este relato de caso foi aprovado pelo comitê de ética e pesquisa (número de parecer 4.622.876) e autorizado pelo paciente e seus responsáveis.

Menino, 12 anos, com sintomas de dor em quadrante superior direito e epigástrio há 20 dias, com piora progressiva, associada à hiporexia há duas semanas e perda de $2 \mathrm{~kg}$ de peso corporal. Não teve vômitos, alteração de hábito intestinal, palidez, sangramento ou febre. Ele eliminava flatos, mas estava sem evacuar por dois dias. Ao exame físico, o paciente era emagrecido, hipocorado, estável hemodinamicamente, mas taquicárdico, com abdome distendido e com uma massa visivel e palpável em epigástrio. O paciente associava o início da dor com uma queda da própria altura durante uma brincadeira.

Ele foi submetido à ultrassonografia de abdome com achado de invaginação ileocecal sem líquido livre na cavidade.

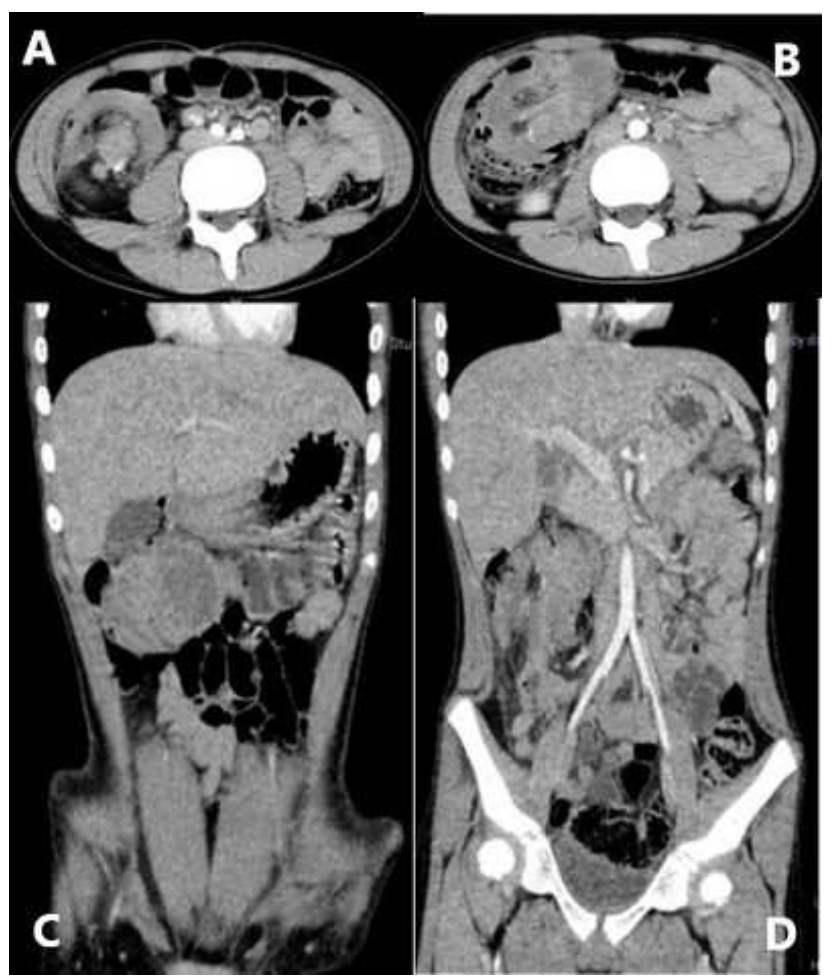

Figura 1. Tomografia de abdome com contraste (fase portal tardia). A. Corte axial evidenciando invaginação ileocecal. B. Corte axial, invaginação extendendo-se para o cólon transverso. C e D. Corte coronal na topografia da invaginação ileocecal, observa-se posição alta da base do ceco.
Apesar de já indicada abordagem cirúrgica, foi optado pela realização de uma tomografia contrastada de abdome para melhor planejamento cirúrgico e investigação etiológica, a qual caracterizou a invaginação íleo-ceco-cólica de $8,5 \mathrm{~cm}$ até a flexura hepática associada a aparente massa hipovascular de 4,2 x 2,8cm, com discretas calcificações de permeio, bem como linfonodos proeminentes de até $10 \mathrm{~mm}$, além de densificação dos planos adiposos (Figura 1).

Foi realizada abordagem por laparoscopia que evidenciou presença de invaginação ileocecal até ângulo hepático, ausência de líquido livre, ausência de lesões peritoneais, mesmo nos recessos e no diafragma. Como as manobras de redução não tiveram exito, foi optado por liberação laparoscópica do cólon direito e transverso, ampliação da incisão umbilical transversa para $6 \mathrm{~cm}$ e exteriorização do segmento acometido. Realizada enterectomia segmentar do íleo terminal e hemicolectomia direita com anastomose látero-lateral ileocólica. A dieta foi iniciada no $2^{\circ}$ dia de pós-operatório e após sete dias de internação, recebeu alta hospitalar.

O estadiamento clínico e o resultado da análise patológica da peça confirmaram o diagnóstico de adenocarcinoma de ceco com $5 \times 4 \mathrm{~cm}$ e acometimento de 3 de 19 linfonodos (T4aN1bM0 Estádio E III B). O subtipo histológico do tumor foi mucinoso invasivo com células em anel de sinete.

Antes do início da quimioterapia, o paciente apresentou um episódio de adenite mesentérica após 21 dias da cirurgia, que foi confirmada por tomografia, tratada com antibiótico, evoluindo bem e sem novas complicações.

Seguindo os protocolos utilizados para pacientes adultos, foi optado pelo início da quimioterapia adjuvante após 4 semanas da cirurgia, com 12 ciclos de quimioterapia, conforme protocolo FOLFOX-6. A colonoscopia realizada após 3 meses foi normal.

O paciente foi submetido à pesquisa genética de mutação no gene p53, prevalente no sul do Brasil, negativa. Não há história familiar de doenças oncológicas nem pólipos intestinais. $\mathrm{O}$ paciente era hígido previamente ao diagnóstico em questão. Devido à ausência de história familiar e patológica pregressa, outras 
pesquisas genéticas não foram acrescentadas.

O paciente manteve-se sem sinais de recidiva locorregional ou à distância da doença, totalizando 14 meses de seguimento. Outras complicações cirúrgicas como hérnia, aderências intestinais, diarreia e dor crônica também não foram observados.

\section{DISCUSSÃO}

A invaginação intestinal é idiopática na maioria dos pacientes pediátricos, sendo que até $90 \%$ das invaginações primárias ocorrem nesta faixa etária 4 . Dentre as causas secundárias, as mais frequentes são Divertículo de Meckel, cistos de duplicação intestinal, Púrpura de Henoch Schonlein, linfomas, pólipos hamartomatosos e adenomegalias $3,5,6$. De $2,2 \%$ a $15 \%$ de todos os pacientes terão algum ponto patológico de invaginação, sendo a regra naquelas acima de 5 anos ${ }^{3}$.

$\mathrm{Na}$ literatura, existem relatos de invaginação por adenocarcinoma de cólon em pacientes adultos ${ }^{4}$. Em pacientes abaixo de 18 anos, é mais comum a invaginação por lesões benignas, pólipos associados a sindromes familiares e metástases ${ }^{7}$. Existe apenas um caso publicado, além deste, de invaginação por adenocarcinoma em paciente adolescente até o momento ${ }^{8}$.

O adenocarcinoma é uma doença rara na população pediátrica. Vundamati D. e colaboradores relataram uma incidência de 1,4 novos casos por $100 \mathrm{mil}$ na faixa etária de 15 a 19 anos, sendo menor ainda a frequência nos menores de 15 anos $^{9}$. Classicamente, os pacientes apresentam subtipos mais agressivos e em estágios mais avançados ${ }^{2}$. Segundo Zhou C. et al, $47 \%$ dos pacientes com até 20 anos de idade se apresentam já com metástase no diagnóstico e $54,3 \%$ dos tumores são mucinosos ou têm células em anel de sinete, sendo assim, duas vezes mais frequente do que em adultos ${ }^{10,11}$. Dados similares também foram observados em outras séries ${ }^{12}$.

A maior parte dos pacientes tem mais de 10 anos $^{2}$ e há um discreto predominio do sexo masculino ${ }^{12,13}$. A localização mais frequente na população pediátrica é em cólon esquerdo (35,7\%), seguida do reto e do cólon direito $(22,9 \%)^{10,11}$. Entretanto, existem divergências na literatura quanto ao predominio da lateralidade ${ }^{9,12}$.

Um levantamento chinês identificou 70 casos $(0,21 \%)$ de adenocarcinoma de cólon em pacientes com idade menor ou igual que 20 anos em nove anos. Em geral, não há associação com doenças familiares e sindromes predisponentes ao câncer colorretal $2,10,11$. Apenas $4,3 \%$ dos pacientes incluídos tinham história familiar de câncer colorretal e 15,3\% de outros tumores malignos ${ }^{10}$. Sabe-se que a presença de instabilidade microssatélite é frequente em pacientes abaixo de 50 anos, correspondendo a $10-15 \%$ dos casos esporádicos ${ }^{11,13}$. Esse fator traz consequências diretas no seguimento a longo prazo destes pacientes.

A colonoscopia faz parte do estadiamento tanto para diagnóstico da doença de base quanto para pesquisa de lesões sincrônicas. A história familiar também é fundamental para investigação de sindromes relacionadas - Síndrome de Peutz-Jeghers, Polipose Familiar Juvenil, Sindrome de Polipose Mista Hereditária, Câncer Colorretal não Polipóide Hereditário e Polipose Adenomatosa Familiar presentes em 3-5\% dos casos $^{2}$. Nestes pacientes, observam-se tumores mais bem diferenciados e relacionados a um adenoma pré-existente 9 .

Dor abdominal, perda de peso e anemia são as principais manifestações, sendo rara a apresentação com sintomas obstrutivos no paciente jovem ${ }^{10,12}$. Todavia, até $50 \%$ dos pacientes procuram atendimento com quadro sugestivo de apendicite aguda $^{2}$. A agressividade intrinseca dos tumores associada ao atraso no diagnóstico têm forte impacto na evolução desses pacientes. No caso relatado, o paciente já apresentava sintomas inespecíficos há quase um mês. Em média, os sintomas de dor e anemia estão presentes por pelo menos há três meses na maioria dos pacientes ${ }^{12-14}$. A lesão do ceco foi a causa da invaginação que levou à obstrução e à agudização dos sintomas. Apesar de relatos na literatura de invaginação por adenocarcinoma em adultos, a invaginação ileocecal por adenocarcinoma de ceco é rara.

O emprego da quimioterapia adjuvante deve ser fortemente considerado mesmo em ressecções completas em pacientes pediátricos, visando uma 
sobrevida livre de doença mais prolongada ${ }^{14}$.

A sobrevida em 3 e 5 anos é baixa, $34,9 \%$ e $23,2 \%$, respectivamente, com tempo médio de sobrevida de 26 meses $^{13}$. O estadiamento é considerado o principal fator prognóstico, uma vez que o risco de morte é 31 vezes maior no estágio IV comparado ao estágio $\mathrm{I}^{1}$. Outros fatores prognósticos estabelecidos são: subtipo histológico mucinoso, ressecção cirúrgica incompleta, presença de mais de $10 \%$ de células em anel de sinete e número de linfonodos avaliados inferior a 1712 . Os protocolos de tratamento se baseiam nos utilizados em adultos, mas a agressividade da doença nos pacientes pediátricos deve ser levada em conta ${ }^{10,11}$.

\section{CONCLUSÃo}

A invaginação intestinal em pacientes acima de cinco anos está fortemente associada a pontos patológicos de invaginação. Causas benignas são mais frequentes, porém, dada a agressividade do adenocarcinoma de cólon nas crianças e nos adolescentes, deve-se estar atento para os sintomas de alarme com alto índice de suspeição, permitindo o diagnóstico precoce e o tratamento oncológico adequado.

\section{REFERÊNCIAS}

1. Surveillance Research Program (SRP) in NCI's Division of Cancer Control and Population Sciences (DCCPS). https://seer.cancer.gov/explorer/appl ica-

tion.html?site $=20 \&$ data_type $=1 \&$ graph -type $=2 \&$ compare

By $=$ age_range $\&$ chk_age_range_16=16

\&sex $=1 \&$ race $=1 \&$ stage $=101 \&$ rate_type

$=2 \& a d v o p t \_p r e c i s i$

on=1\&advopt_show_ci=on\&advopt_dis play $=1$ (acesso em 04/10/2020 as 20h).

2. Al-Tonbary Y, Darwish A, El-Hussein A, Fouda A. Adenocarcinoma of the colon in children: Case series and mini-review of the literature. Hematol Oncol Stem Cell Ther. 2013;6(1):2933. doi:10.1016/j.hemonc.2013.02.003.

3. Zhao L, Feng S, Wu P, Lai X, Lv C, Chen G. Clinical characteristics and surgical outcome in children with in- tussusceptions secondary to pathologic lead points: retrospective study in a single institution. Pediatr Surg Int. 2019;35(7):807-11.

doi: $10.1007 / \mathrm{s} 00383-019-04471-8$.

4. Fan WF, Ma G, Li GC, Long J, Xu YH, Guo KJ, et al. Ileocecal intussusception caused by two different tumors which is the culprit lesion? A case report. World J Clin Cases. 2020;8(10): 2044-9.

doi: 10.12998/wjcc.v8.i10.2044.

5. Ondhia MN, Al-Mutawa Y, Harave S, Losty PD. Intussusception: A 14-year experience at a UK tertiary referral centre. J Pediatr Surg. 2020;55(8):1570-3.

doi: 10.1016/j.jpedsurg.2019.07.022.

6. Kolar M, Pilkington M, Winthrop A, Theivendram A, Lajkosz K, Broglyac SB. Diagnosis and treatment of childhood intussusception from 1997 to 2016: A population-based study. J Pediatr Surg. 2020;55(8):1562-9.

doi: 10.1016/j.jpedsurg.2020.01.049.

7. Di Buono G, Randisi B, Romano G, Ricupati F, Buscemi S, Agrusa A. Recurrent intussusception of small bow$\mathrm{el}$ in a young patient due to metastases from cardiac undifferentiated pleomorphic sarcoma: A first ever case report. Int $J$ Surg Case Rep. 2020;77S(Supp1):S13-S16.

doi: 10.1016/j.ijscr.2020.09.065.

8. Tarabishi AS, Aljarad Z, Shebli B, Masri AH, Anadani R, Shabouk MB, et al. A rare case of bowel intussusception due to adenocarcinomatous polyp in a 14 year old child: case report. BMC Surg. 2020;20(1):198.

doi: 10.1186/s12893-020-00859-9.

9. Vundamati DS, Chen X, Singh V. Colonic Adenocarcinoma at Advanced Stage in Adolescence: Report of 2 Cases. Case Rep Pathol. 2020:1848367. doi: $10.1155 / 2020 / 1848367$.

10. Zhou C, Xiao W, Wang X, Chen H, Niu S, Wang Q, et al. Colorectal cancer under 20 years old: a retrospective analysis from three tertiary hospitals. $\mathrm{J}$ Cancer Res Clin Oncol. 2021;147(4):1145-55.

doi: $10.1007 / \mathrm{s} 00432-020-03397-2$.

11. Poles GC, Clark DE, Mayo SW, Beierle EA, Goldfarb M, Gow KW, et al. Colorectal carcinoma in pediatric patients: 
A comparison with adult tumors, treatment and outcomes from the $\mathrm{Na}$ tional Cancer Database. J Pediatr Surg. 2016;51(7):1061-6.

doi: $10.1016 /$ j.jpedsurg.2015.11.005.

12. Hill DA, Furman WL, Billups CA, Riedley SE, Cain AM, Rao BN, et al. Colorectal Carcinoma in Childhood and Adolescence: A Clinicopathologic Review. J Clin Oncol. 2007;25(36):5808-14.

doi: 10.1200/JCO.2007.12.6102.

13. Du F, Shi SS, Sun YK, Wang JW, Chi Y. Clinicopathological Characteristics and Prognosis of Colorectal Cancer in Chinese Adolescent Patients. Chin Med J (Engl). 2015;128(23):3149-52. doi: 10.4103/0366-6999.170256.

14. Goldberg J, Furman WL. Management of Colorectal Carcinoma in Children and Young Adults. J Pediatr Hematol Oncol. 2012;34(Suppl2):S76-9. doi: 10.1097/MPH.0b013e31824e38c1.
Fonte de financiamento: Não

Conflito de interesses: Não

Data de Submissão: 07 Abril 2021

Decisão final: 30 Agosto 2021

Autor de Correspondência:

Elisângela de Mattos e Silva

E-mail: elismattos@gmail.com 